\title{
Artigos Publicados em Periódicos Brasileiros de Interesse para a Medicina do Exercício e do Esporte. Uma Revisão.
}

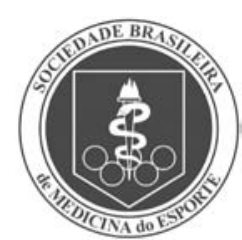

Artigos de Revisão

\section{Articles Published in Brazilian Journals Relevant to Sports and Exercise Medicine. A Review}

Arnaldo José Hernandez Editor-Chefe, Rev Bras Med Esporte

E-mail: ajhernandez@uol.com.br

Submetido em: 29/09/2009 Versão final recebida em: 14/10/2009 Aceito em: 29/10/2009

\section{RESUMO}

Esta nova série de artigos tem por objetivo levar aos leitores nacionais e internacionais algumas das mais importantes contribuições provenientes da literatura médica brasileira recente. Embora possa parecer que não tenham relação direta com a Medicina do Exercício e do Esporte, são trabalhos que podem oferecer suporte a muitas linhas de pesquisa nessa área. Os artigos originais mais relevantes são selecionados por experientes editores, a quem solicitamos que escolham palavras- chave para que sejam destacadas para chamar a atenção do leitor. Para facilitar a leitura, os artigos são organizados por área de interesse. Para aproveitar ao máximo o limitado espaço editorial, não são incluídos os nomes dos autores dos artigos. Entretanto, a referência completa é oferecida ao final do artigo. O resultado final traz o que há de melhor do artigo, seguido de uma sintética interpretação pessoal. Endereçado ao médico ocupado, esperamos que esta inciativa possa contribuir para o sucesso da translação do conhecimento da evidência científica para a prática clínica.

Palavras-chave: medicina esportiva, cardiologia, ortopedia, nutrição.

\begin{abstract}
This brand-new series of articles aims at delivering to national and international readers some of the cutting-edge contributions from the Brazilian medical literature. Some of them may not be directly related to the area, but they can be the grounding for scientific research in the field of Sports and Exercise Medicine. Recently, papers published in the main Brazilian medical journals are carefully selected and analyzed by skilled medical editors. In addition, we asked editors to choose keywords to be highlighted in order to call the reader's attention. Articles are organized by area of interest to facilitate reading. To get the most of the limited available editorial space we did not include the names of the authors of the related articles in the text itself, but a complete reference guide is provided at the end of the article. The result carries the best from the original paper accompanied by a personal interpretation. Directed to the busy medical doctor, we hope that this initiative may contribute to the successful translation of knowledge from scientific evidence to clinical practice.
\end{abstract}

Keywords: sports medicine, cardiology, orthopedics, nutrition.

\section{MEDICINA DO ESPORTE}

Foram selecionados 22 artigos diretamente relacionados com Medicina do Exercício e do Esporte. Os parâmetros cardíacos, eletrocardiográficos e funcionais, e respostas cardiopulmonares ao exercício em corredores brasileiros de longa distância, caracterizam-se por bradicardia de repouso e hipertrofia ventricular esquerda ${ }^{(1)}$. Estudos realizados em jogadores de futebol mostraram que o consumo máximo de oxigênio $\left(\mathrm{VO}_{2 \max }\right)$ em 11 futebolistas masculinos de alto nível avaliado por duas vezes, com intervalo de 15 dias entre os testes, se mostrou reprodutível; houve correlação significante e alta do $\mathrm{VO}_{2 \max }$ entre os dois testes, mostrando que o protocolo de Heck modificado é adequado para essa avaliação(2). Mostraram também que a suplementação alimentar com carboidrato e glutamina mostrou ser mais eficiente que a suplementação simples com carboidratos em termos de tempo de corrida, distância coberta e sensação de fadiga em futebolistas de alto nível atlético ${ }^{(3)}$.

A incidência de lesões musculoesqueléticas em atletas de elite do basquetebol feminino foi analisada prospectivamente e revelou predominância de lesões leves e a região do joelho foi a de maior morbidade ${ }^{(4)}$.

A tolerância ao exercício de crianças e adolescentes submetidos a teste ergométrico em esteira foi medida com os protocolos de Bruce ou em rampa; verificou-se que a velocidade e a inclinação alcançadas com o protocolo em rampa podem ser utilizadas como referência para auxiliar na orientação do exercício. O protocolo em rampa mostrouse superior ao de Bruce ${ }^{(5)}$. Por outro lado, a pressão arterial sistólica e diastólica em adolescentes submetidos ao teste de esforço foi medida no repouso, no esforço máximo e aos seis minutos da recuperação. Durante o exercício a pressão sistólica teve relação direta com idade, peso, altura e índice de massa corpórea do indivíduo; a diastólica teve relação apenas com a idade ${ }^{(6)}$.

Vacanti et al. verificaram que o teste ergométrico é factível, eficaz e custo-efetivo na predição de eventos cardiovasculares no paciente muito idoso, quando comparado com a cintilografia de perfusão miocárdica(7). A frequência cardíaca máxima medida durante um teste de esforço progressivo foi comparada, em idosas brasileiras, com valores estimados pelas equações "220 - idade" e a de Tanaka et al., observando-se que ambas as equações superestimam significativamente os valores obtidos ${ }^{(8)}$.

Battaglini et al. verificaram que exercícios com treinamento de força promovem mudanças benéficas na composição corporal e força em pacientes portadoras de câncer de mama sob tratamento médico ${ }^{(9)}$.

Zanetti et al. mostraram que o acompanhamento fisioterapêutico proporcionou melhores resultados subjetivos e objetivos no tratamento da incontinência urinária de esforço feminina pela cinesioterapia do assoalho pélvico(10). Por outro lado os efeitos da cinesioterapia nos músculos do assoalho pélvico revelaram aumento significativo na pressão e na força durante a gestação. Durante o período gestacional houve correlação positiva e significativa entre a avaliação funcional do assoalho pélvico e as perineometrias ${ }^{(11)}$.

O treinamento físico associado com BiPAP ${ }^{\circledR}$ aumenta a capacidade muscular oxidativa e pode ser um recurso coadjuvante da reabilitação física de pacientes com doença pulmonar obstrutiva crônica ${ }^{(12)}$. 
Num modelo experimental, o bloqueio a curto prazo da síntese de NO, em animais sedentários, induziu hipertensão, sem hipertrofia cardíaca. Em animais treinados atenuou a hipertensão e promoveu hipertrofia cardíaca com aumento da fibrose miocárdica, sugerindo importante papel do NO nas adaptações teciduais cardíacas induzidas pelo treinamento físico aeróbio ${ }^{(13)}$.

A prática de atividade física na fase pré-operatória resultou em tempo de internação hospitalar mais curto e menor número de complicações trans e pós-operatórias no período de um ano. A cirurgia cardíaca aumentou o número de pacientes fisicamente ativos no seguimento de um ano(14).

Issa et al. mostraram que o bisoprolol produziu melhora clínica e hemodinâmica e de função cardíaca nas diferentes etiologias, sem, entretanto, apresentar efeitos de melhora na capacidade de exercício(15).

Garbelotti Júnior et al. propuseram uma técnica de baixo custo e de fácil reprodutibilidade para o estudo biomecânico em cadáveres. A técnica foi eficiente ao demonstrar a deformação plástica dos ligamentos cruzados. Os resultados podem auxiliar no planejamento de programas de reabilitação física ${ }^{(16)}$.

Na reconstrução do ligamento cruzado anterior, o não retorno ao mesmo nível funcional pré-lesão não pode ser explicado pela frouxidão ligamentar residual ou pelas diferenças do desempenho muscular e funcional ${ }^{(17)}$.

A análise da translação anterior da tíbia, pico de torque e atividade eletromiográfica do quadríceps e isquiotibiais em indivíduos com lesão do ligamento cruzado anterior em cadeia cinética aberta mostra que indivíduos com lesão do LCA e indivíduos controle se comportam de maneira similar em relação a pico de torque isométrico e controle motor, embora a artrocinemática da articulação tibiofemoral tenha se mostrado diferente em joelhos lesados ${ }^{(18)}$.

Em lesões associadas à lesão do ligamento cruzado anterior, embora haja tendência de aumento de lesões meniscais após seis meses e de lesões condrais após 12 meses da lesão do ligamento cruzado anterior, tal fato não mostrou significância estatistica ${ }^{(19)}$. A frouxidão do ligamento cruzado anterior não alterou significantemente o pico de torque dos flexores e extensores e a relação ísquiotibiais/quadríceps dos joelhos de atletas corredores de longas distâncias ${ }^{(20)}$.

Barbosa e Gonçalves analisaram a fadiga de músculos lombares e as variáveis demográficas relacionadas com ela. Intervenções destinadas ao tratamento de problemas na coluna lombar devem considerar os vários fatores responsáveis pela fadiga dos músculos dessa região(21).

Kirkwood et al. analisaram a biomecânica das artifculações do quadril e dos joelhos durante diferentes atividades e concluíram que essa análise é essencial para melhor compreensão do papel de cada articulação durante o movimento, contribuindo assim para a elaboração de melhores programas físicos de reabilitação, prevenção e treinamento de performance $e^{(22)}$.

\section{CARDIOLOGIA}

Foram selecionados 17 artigos cardiológicos de interesse para a Medicina do Exercício e do Esporte. Ângelo et al. determinaram valores do percentil de 95\% do septo interventricular e da parede posterior e, consequentemente, de massa ventricular esquerda absoluta e indexada na população de Vitória, e verificaram que são inferiores aos valores encontrados em estudos prévios. Nesse aspecto, os resultados desse estudo serão úteis como referência, pois estão de acordo com os novos limites sugeridos na literatura para o diagnóstico ecocardiográfico de hipertrofia ventricular esquerda ${ }^{(23)}$. Por outro lado, o ecocardiograma demonstrou boa funcionalidade do aparelho valvar mitral nos pacientes submetidos ao implante das próteses para substituição de cordas tendíneas rotas ou alongadas e afiladas com adequada coaptação das cúspides ${ }^{(24)}$. A estimulação atrial direita temporária reduziu a incidência de fibrilação atrial pós-operatória. A idade avançada e a não estimulação atrial foram fatores preditivos independentes para a ocorrência dessa arritmia ${ }^{(25)}$. A plástica da valva mitral com a técnica do "duplo Teflon" mostrou baixa morbimortalidade e boa evolução clínica tardia(26). As estratégias de uso de antifibrinolíticos, hemodiluição normovolêmica e reposição total do perfusato reduziram a necessidade de hemotransfusão no pósoperatório de cirurgia cardíaca(27). Takiuti et al. avaliaram a qualidade de vida (QV) na doença multiarterial coronariana em pacientes submetidos randomicamente a cirurgia, angioplastia ou tratamento clínico, e observaram melhora em todos os domínios e nas três opções terapêuticas. Comparativamente, a cirurgia ofereceu melhor qualidade de vida após quatro anos de seguimento ${ }^{(28)}$. Mion et al. avaliaram os procedimentos adotados pelos médicos brasileiros para o diagnóstico e tratamento da hipertensão em relação aos preconizados pelas IV Diretrizes Brasileiras de Hipertensão e concluíram que o seguimento nas diretrizes é apenas parcial. Questões como melhor distribuição do documento, normatização de valores para diagnóstico e alvo terapêutico e formas de abordar o paciente hipertenso dentro de seu risco cardiovascular global deverão ser mais bem abordadas quando da confecção de futuras diretrizes ${ }^{(29)}$. Cavalcante et al. compararam aspectos relacionados com a QV de pacientes hipertensos em tratamento: dados clínicos e sociodemográficos com enfoque na sexualidade, autopercepção da QV, número e tipo de medicamentos utilizados e sua interferência na vida sexual. Foi aplicado o questionário SF-36, que não detectou modificações na QV entre os grupos, por tratar-se de doença crônica assintomática ${ }^{(30)}$.

A reeducação respiratória com a técnica de respiração lenta mostrou ser um bom recurso complementar para o controle tanto cardiovascular como respiratório em pacientes hipertensos ${ }^{(31)}$

A massa ventricular esquerda do coração dos homens foi superior ao relatado na literatura contemporânea, sugerindo que a presença de hipertrofia de VE pode ser inferida em presença de massa de VE superior a $218 \mathrm{~g}$ ou $128 \mathrm{~g} / \mathrm{m}$ nos homens e $148 \mathrm{~g}$ ou $88 \mathrm{~g} / \mathrm{m}$ nas mulheres ${ }^{(32)}$. Já a pressão de pulso obtida por monitorização residencial da pressão arterial e sua relação com o índice de massa do ventrículo esquerdo. De Marco et al. relatam resultados de monitorização residencial de pressão arterial, sem correlação entre a PAD e o IMVE. A PAS e a PP correlacionam-se positivamente com o IMVE ${ }^{(33)}$.

Machado et al. caracterizaram cuidadores de candidatos a transplante do coração e identificaram um familiar (cônjuge), do sexo feminino, com idade média de 44,3 anos, renda própria, classificados, na maioria, como "bons" ou "regulares", não havendo correlação com escolaridade, atividade profissional e renda ${ }^{(34)}$.

A cardioplegia sanguínea contínua e tépida foi mais eficiente na preservação da integridade estrutural e ultraestrutural do miocárdio, quando comparada com a cardioplegia cristaloide intermitente e fria(35).

Após o infarto do miocárdio, num modelo experimental, o tabagismo e o betacaroteno promoveram intensificação do processo de remodelação cardíaca; houve potencialização dos efeitos deletérios no processo de remodelação com os dois tratamentos em conjunto ${ }^{(36)}$.

Alguns modelos foram desenvolvidos. A bomba sanguínea espiral mostrou resolutividade por gerar escoamento e pressão adequados, sem causar danos excessivos aos elementos figurados do sangue ${ }^{(37)}$. Um modelo de coração isolado desenvolvido tipo working heart se manteve estável durante a duração dos experimentos, sem a administração de drogas cardiotônicas, e possibilitou a mensuração de todos os dados propostos de forma efetiva com o coração executando trabalho(38) A baixa incidência de eventos adversos em pacientes portadores de cardioversor-desfibrilador implantável e de morte súbita sugere ser essa uma estratégia terapêutica valiosa no manejo de pacientes isquêmicos e não isquêmicos selecionados previamente ${ }^{(39)}$.

\section{ORTOPEDIA}

Foram identificados 10 artigos de interesse puramente ortopédico com significância para Medicina do Exercício e do Esporte. Os genes do receptor da vitamina D e da glutatião transferases $\mathrm{M} 1$ e T1, bem como o hábito de fumar, são fatores genéticos e ambientais envolvidos na degeneração do disco intervertebral ${ }^{(40)}$. Oliveira et al. concluíram que as fibras nervosas do tipo III são mais frequentes na região anterior; as fibras 
dos tipos II e IV são mais frequentes na região posterior; e as fibras do tipo I não apresentaram diferenças entre a região anterior e a posterior; além disso, o disco degenerado tem mais fibras nervosas que o disco normal(41). Demange et al. estudaram o ligamento da cabeça do fêmur e concluíram que o mesmo influi limitando a adução do quadri|(42).

Os ligamentos do joelho foram extensamente estudados. Lesões do ligamento cruzado anterior associadas às de outros ligamentos, exceto o posterior, associam-se à prática de esportes e quedas. Lesões do ligamento cruzado posterior associam-se a acidentes de tráfego e impactos diretos sobre o joelho ${ }^{(43)}$. Alterações de distribuição histoanatômica de colágeno tipo I, III e V promovem remodelamento adaptativo em consequência de ruptura do tendão tibial posterior ${ }^{(44)}$

O uso de medicamentos pode ser considerado como fator de risco para fratura por queda. Os resultados também podem servir como orientação para equipe médica, pacientes e seus familiares, no sentido de tentar evitar quedas, principalmente quando o uso de medicamentos é necessário ${ }^{(45)}$.

Belloti et al. verificaram que a conduta dos ortopedistas brasileiros quanto aos principais aspectos do manejo das fraturas de Colles é concordante quanto à forma de tratamento conservador e à utilização de enxerto ósseo. Há conflito de opiniões quanto ao método de classificação das fraturas; aos métodos de tratamento cirúrgico e às complicações ${ }^{(46)}$.

Fernandes et al. mostraram que dores nas costas são mais intensas e determinaram maior limitação das atividades habituais em pacientes portadoras de hipertrofia mamária ${ }^{(47)}$.

A qualidade de vida e a função pós-operatória em pacientes submetidos à artroplastia de Girdlestone, na população brasileira, ainda necessitam ser mais pesquisadas, pois os resultados são indicações do comportamento das variáveis de estudo e não podem ser consideradas encerradas (48).

A versão em português do Questionário de Claudicação de Edimburgo manteve níveis adequados de sensibilidade e especificidade, podendo ser recomendado para o rastreamento de doença arterial obstrutiva na prática clínica e em estudos epidemiológicos realizados no Brasil(49).

\section{NUTRIÇÃO}

Quatorze artigos originais sobre aspectos nutricionais apresentam interesse direto para a Medicina do Exercício e do Esporte. A síndrome metabólica (SM) está fortemente associada à presença de insuficiência cardíaca com função sistólica preservada e ao sexo feminino em nossa comunidade ${ }^{(50)}$. Adicionalmente, a síndrome metabólica representa um potencial problema de saúde pública, particularmente em mulheres e indivíduos com sobrepeso na área rural estudada. O sobrepeso, a idade acima de 60 anos e o sexo feminino foram considerados fatores independentes associados a essa condição(51). Um perfil lipídico alterado, em homens entre 31 e 50 anos, confere maior risco para doenças cardiovasculares em adulto jovem, enquanto padrão semelhante nas mulheres, após essa faixa etária, pode refletir alterações hormonais fisiológicas. Ambos os critérios para diagnóstico de SM distinguem pacientes de controles, destacando-se IDF, com parâmetros, no entanto, nem sempre associados a alto risco. Alta prevalência de SM em pacientes, mesmo com acompanhamento cardiológico, sugere predisposição para manifestações cardiovasculares em indivíduos brasileiros ${ }^{(52)}$.

Karnikowski et al. observaram que a frequência de esteatose hepática não alcoólica idiopática em brasileiros idosos e de meia-idade é maior que a descrita em outros estudos. Os achados sugerem que metabolismo glicêmico anormal conjugado a maior aporte lipídico e/ou biossíntese exacerbada no fígado são prováveis mecanismos fisiopatogênicos da esteatose hepática não alcoólica na população estudada(53).

Segundo Greve et al., um alto índice de massa corpórea exige maior deslocamento corporal para manter o equilíbrio postural(54). Fernandes et al. avaliaram o desempenho de diferentes valores críticos de índice de massa corporal na identificação de excesso de gordura corporal e obesidade abdominal em adolescentes e concluíram que todos convergem na indicação do estado nutricional e da obesidade abdominal. No entanto, um índice defendido nesse estudo mostrou-se mais sensível na indicação de indivíduos obesos com elevado risco cardiovascular ${ }^{(55)}$. Por outro lado, medidas disponíveis para a população dos Estados Unidos para avaliação de circunferência de cintura mostraram baixa sensitividade e especificidade para hipertensão na população brasileira. O índice de massa corpórea também mostrou baixa sensitividade ${ }^{(56)}$. Araújo et al. mostraram que em mulheres e homens sem nenhum indício de cardiopatia, a relação colesterol total/ HDL-colesterol aumentou com o índice de massa corpórea em ambos os sexos. Outros índices associados ao índice de massa corpórea foram glicose sérica nas mulheres e idade nos homens. As variáveis clínicas e laboratoriais associadas ao índice de massa corporal podem diferir quanto ao sexo ${ }^{(57)}$. Nunes et al. mostraram elevadas frequências de sobrepeso e obesidade entre adolescentes em Campina Grande/PB, assim como o tempo gasto assistindo a TV e os maus hábitos alimentares, especialmente nos pertencentes às classes econômicas mais favorecidas. É reduzido o tempo despendido com atividades físicas de lazer, principalmente nas classes econômicas desfavorecidas ${ }^{(58)}$. A prevalência do excesso de peso e sobrepeso em pré-escolares na cidade de Natal é alta, principalmente nas escolas privadas, demonstrando a necessidade de implantação de programas de prevenção e intervenção, a partir da educação infanti|(59). No entanto, numa população de obesos, o excesso de peso não provocou alterações na força dos músculos respiratórios e as modificações não foram influenciadas pela distribuição de gordura predominante em porção superior corporal(60).

O ecocardiograma realizado em obesos graves assintomáticos revelou alterações cardíacas estruturais comuns na miocardiopatia da obesidade, que podem associar-se ao desenvolvimento de insuficiência cardíaca, arritmias e morte súbita, possibilitando a identificação de pacientes sob maior risco cardiovascular ${ }^{(61)}$.

Barbosa et al. mostraram associação entre estado nutricional e capacidade funcional para as mulheres, sendo a obesidade a condição nutricional limitante ao bom desempenho nos testes. Sexo e grupo etário devem ser considerados na associação entre estado nutricional e o desempenho motor ${ }^{(62)}$.

Guedes et al. indicam que intervenções direcionadas à adoção de um estilo de vida saudável, incluindo abstenção do uso de tabaco, deverão iniciar-se em idades precoces na tentativa de prevenir ou retardar o desenvolvimento de lesões ateroscleróticas e minimizar o aparecimento de coronariopatias prematuras na idade adulta(63).

\section{TEMAS GERAIS}

Nove estudos gerais apresentam interesse para a Medicina do Exercício e do Esporte. A recente publicação de artigo com estatísticas do Banco de Dados do Sistema Único de Saúde (Datasus) mostrou que os resultados de cirurgia cardiovascular em pacientes operados em hospitais do Sistema Único de Saúde (SUS), entre janeiro de 2000 e dezembro de 2003, podem não ser equivalentes aos dos Estados Unidos e Reino Unido. Os resultados de mortalidade após cirurgia cardiovascular realizada em hospitais do SUS no Brasil estariam ao redor de $8 \%$. Esses dados foram comparados com os do banco de dados da Sociedade de Cirurgiões Torácicos dos EUA (STS - The Society for Thoracic Surgeons) e com os Registros de Cirurgia Cardíaca do Reino Unido (UK Cardiac Surgical Register), que estariam em torno de $4 \%{ }^{(64)}$. O comércio de trabalhos científicos é uma realidade que pode interferir negativamente na formação ética, científica e profissional de graduandos e pós-graduandos, bem como na produção científica, falseando dados e informações da literatura. Recomenda-se uma nova abordagem, principalmente na avaliação de trabalhos de conclusão de cursos e monografias ${ }^{(65)}$.

Galvão et al. verificaram grande interesse e atitude positiva dos estudantes de medicina sobre doação e transplante de órgãos, embora a maioria tenha declarado conhecimento deficiente sobre o tema. Observaram também atitude negativa dos estudantes do quarto, quinto e sexto ano médico em relação à doação para alcoólatras, não doadores, usuários de drogas ilícitas, estrangeiros e criminosos ${ }^{(66)}$. 
Um retrato das mortes por causas externas no Estado de São Paulo indica a necessidade de desenvolver estratégias para a prevenção do problema, que devem levar em conta as diferenças existentes entre as faixas etárias no que diz respeito à intencionalidade e mecanismo da lesão. Especial atenção deve ser dada aos homicídios e aos acidentes de transporte ${ }^{(67)}$.

Pugliese et al. relatam que o tratamento farmacológico aliado a um programa de intervenção psicológica destinado a reduzir o nível de estresse e mudar o comportamento alimentar resultou em benefícios adicionais na redução de risco coronariano(68).

Reduções na capacidade vital, do volume corrente e do volume minuto pulmonar foram observadas durante o período pós-operatório em pacientes submetidos a clipagem de aneurisma ou ressecção de tumor. A redução na capacidade vital e no volume corrente foram maiores em pacientes submetidos a craniotomia por aneurisma com tempo cirúrgico mais longo ${ }^{(69)}$.

Maciel et al. caracterizaram os diferentes componentes da acidose metabólica de pacientes com hiperlactatemia de modo a verificar o quanto o lactato é responsável pela acidose e a relevância que isso possa ter no prognóstico desses pacientes. Hiperlactatemia é comumente acompanhada de acidemia metabólica, porém o lactato corresponde a uma parcela minoritária da acidose; ânions não mensuráveis contribuem com a maior parte da carga ácida em pacientes hiperlactatêmicos. O percentual da acidose devido à hiperlactatemia não foi relevante em termos de prognóstico ${ }^{70}$.

Moser et al. verificaram que relacionamento familiar saudável e religiosidade estão associados à conduta de sexo seguro. É relevante a percentagem de estudantes que ainda têm conduta de sexo inseguro, mostrando que ser universitária e frequentar cursos de ciências da saúde não são garantia de comportamento sexual seguro(71).

Antonello et al. verificaram que a natriúria de 24 horas foi maior em indivíduos com limiar de sensibilidade gustativa ao sal aumentado, sugerindo que essa maior avidez ao sal determine maior ingestão de sódio. Houve forte associação entre pressão arterial e índice de massa cardíaca. Não foi evidenciada associação entre limiar de sensibilidade gustativa ao sal e pressão arterial, diferente do já documentado em pacientes com pressão arterial aumentada ${ }^{(72)}$.

O autor declara não haver qualquer potencial conflito de interesses referente a este artigo.

\section{REFERÊNCIAS BIBLIOGRÁFICAS}

Azevedo LF, Brum PC, Rosemblatt D, Perlingeiro PS, Barretto ACP, Negrão CE, et al. Características cardíacas e metabólicas de corredores de longa distância do ambulatório de cardiologia do esporte e exercício, de um hospital terciário. Arq Bra. Cardiol. 2007:88:17-25

2. Santos-Silva PR, Fonseca AJ, Castro AW, Greve JMD, Hernandez AJ. Reprodutibilidade da potência aeróbia máxima $\left(\mathrm{VO}_{2 \text { max }}\right.$ ) em jogadores de futebol utilizando o protocolo de Heck modificado. Clinics. 2007;62:391-6.

jogadores de futebol utilizando o protocolo de Heck modificado. Clinics. 2007;62:391-6. 3. Favano A, Santos-Silva PR, Nakano EY, Pedrinelli A, Hernandez AJ, Greve JMD. A suplementar

4. Silva AS, Abdalla RJ, Fisberg M. Incidência de lesöes musculoesqueléticas em atletas de elite do basquetebol feminino. Acta Ortop. Bras. 2007;15:43-6

Silva OB, Saraiva LCR, Sobral Filho D. Teste ergométrico em crianças e adolescentes: maior tolerância ao esforço com o protocolo em rampa. Arq Bras Cardiol. 2007;89:391-7.

Becker MMC, Silva OB, Moreira IEG, Victor EG. Pressăo arterial em adolescentes durante teste ergométrico. Arq Bras Cardio. 2007;88:329-33.

Vacanti $\sqcup$, Sposito AC, Séspedes L, Sarpi M, Ramires JAF, Bortnick AE. O teste ergométrico é factível, eficaz e custo-efetivo na predição de eventos cardiovasculares no paciente muito idoso, quando comparado à cintilografia de perfusão miocárdica. Arq Bras Cardiol. 2007;88:531-6.

8. Silva VAP, Bottaro M, Justino MA, Ribeiro MM, Lima RM, Oliveira RJ. Freqüência cardíaca máxima em idosas brasileiras: uma comparaçăo entre valores medidos e previstos. Arq Bras Cardiol. 2007;88:314-20.

Battaglini C, Bottaro M, Dennehy C, Rae L, Shields E, Kirk D, et al. Os efeitos da prescrição individualizada de exercícios na com-

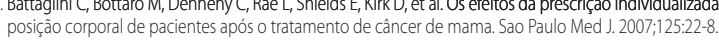

posição corporal de pacientes após o tratamento de câncer de mama. Sao Paulo Med J. 2007;125:22-8.
10. Zanetti MRD, Castro RA, Rotta AL, Santos PD, Sartori M, Girão MJBC. Impacto da supervisão fisioterapêutica aos exercícios do

assoalho pélvico para tratamento da incontinência urinária de esforço. Sao Paulo Med I. 2007:25:265-269.

11. Oliveira C, Lopes MAB, Pereira LCL, Zugaib M. Efeitos da cinesioterapia no assoalho pélvico durante a gravidez. Clinics. 2007;62:439-46.

12. Toledo A, Borghi-Silva A, Sampaio LMM, Ribeiro KP, Baldissera V, Costa D.

13. Souza HCD, Penteado DMD, Martin-Pinge MC, Barbosa Neto O, Teixeira VPA, Blanco JHD, et al.

4. Nery RM, Barbisan JN, Mahmud MI. Influência da prática da atividade física no resultado da cirurgia de revascularização mio-

cárdica. Rev Bras Cir Cardiovasc. 2007;22:297-302.

Issa VS, Guimarães GV, Rezende MVC, Cruz FD, Ferreira SMA, Bacal F, et al . Efeito do bisoprolol sobre a função cardíaca e exercício em pacientes com insuficiência cardíaca. Arq Bras Cardiol. 2007;88:340-5.

16. Garbelotti Júnior SA, Pelozo Júnior O, Caldana RP, Ramalho Jr A, Smith RL. Avaliação experimental do comportamento cinemático tridimensional dos ligamentos cruzados. Clinics. 2007:62:619-26.

17. Lustosa LP, Fonseca ST, Andrade MAP. Reconstrução do ligamento cruzado anterior: impacto do desempenho muscular e funcional no retorno ao mesmo nível de atividade pré-lesão. Acta Ortop Bras. 2007;15:280-4.

18. Vasconcelos RA, Arakaki J, Coelho, SAP, Oliveira AS, Paccola CJ, Bevilaqua-Grossi D. Análise da translação anterior da tíbia, pico de torque e atividade eletromiográfica do quadríceps e isquiotibiais em indivíduos com lesão do ligamento cruzado anterior em cadeia cinética aberta. Acta Ortop Bras. 2007:15:14-8.

19. Rocha ID, Moraes TMS, Rezende MU, Pécora. Avaliaçăo da evolução de lesões associadas à lesăo do ligamento cruzado anterior. Acta Ortop Bras. 2007;15:105-8.

Acta Ortop Bras. 2007;15:105-8.
20. Portes EM, Portes LA, Botelho VG, Souza-Pinto S. Pico de torque isocinético e relação ísquios-tibiais/quadríceps em atletas de

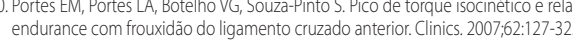

endurance com frouxidáo do ligamento cruzado anterior. Cinics. $2007 ; 62: 127-32$.
Barbosa FSS, Gonçalves M. A proposta biomecânica para a avaliaçăo de sobrecarga na coluna lombar: efeito de diferentes variáveis demográficas na fadiga muscular. Acta Ortop Bras. 2007;15:132-7.

2. Kirkwood RN, Gomes HA, Sampaio RF, Culham E, Costigan P. Análise biomecânica das articulações do quadril e joelho durante a marcha em participantes idosos. Acta Ortop Bras. 2007;15:267-71.

23. Ângelo LCS, Vieira MLC, Rodrigues SL, Morelato RL, Pereira AC., Mill JG, et al. Valores de referência de medidas ecocardiográficas em amostra da população brasileira adulta assintomática. Ara Bras Cardiol. 2007;89:184-90

24. Leal JC, Gregori Jr F, Galina LE, Thevenard RS, Braile DM. Avaliação ecocardiográfica em pacientes submetidos à substituiçăo de cordas tendíneas rotas, Rev Bras Cir Cardiovasc 2007:22:184-91.

25. Avila Neto V, Costa R, Silva KR, Martins ALM, Moreira LFP, Santos LB, et al. Efeitos da estimulaçăo temporária atrial direita na prevenção da fibrilação atrial no pós-operatório de revascularizaçăo do miocárdio com circulação extracorpórea. Rev Bras $\mathrm{Ci}$ Cardiovasc. 2007;22:332-40

26. Brandão CMA, Guedes MAV, Silva ME, Vieira ML, Pomerantzeff PMA, Stolf NAG. Plastia da valva mitral com a técnica do "Duplo Teflon": resultados de 10 anos. Rev Bras Cir Cardiovasc. 2007;22:448-53.

27. Souza HJB, Moitinho RF. Estratégias para reduçăo do uso de hemoderivados em cirurgia cardiovascular. Rev Bras Cir Cardiovasc. 2008; $23: 53-9$.

28. As estratégias de uso de antifibrinolíticos, hemodiluição normovolêmica e reposiçăo total do perfusato reduziram a necessidad de hemotransfusăo no pós-operatório de cirurgia cardíaca.

9. Takiuti ME, Hueb W, Hiscock SB, Nogueira CRSR, Girardi P, Fernandes F, et al . Qualidade de vida após revascularizaçăo cirúrgic do miocárdio, angioplastia ou tratamento clínico. Arq Bras Cardiol. 2007;88: 537-44.

30. Mion Junior D, Silva GV, Gusmão JL, Machado CA, Amodeo C, Nobre F, et al. Os médicos brasileiros seguem as diretrizes brasileiras de hipertensão? Arq Bras Cardiol. 2007;88:212-7.

31. Cavalcante MA, Bombig MTN, Luna Filho B, Carvalho ACC, Paola AAV, Póvoa R. Qualidade de vida de pacientes hipertensos em tratamento ambulatorial. Arq Bras Cardiol. 2007:89:245-50

32. Pinheiro CHJ, Medeiros RAR, Pinheiro DGM, Marinho MJF. Modificação do padrăo respiratório melhora o controle cardiovascular na hipertensão essencial. Arq Bras Cardiol. 2007;88:651-9.

33. Rodrigues SL, Pimentel EB, Mill JG. Peso dos ventrículos cardíacos determinado em necropsia de indivíduos saudáveis mortos por causas externas. Arq Bras Cardiol. 2007;89:279-84.

por causas externas. Arq Bras Cardiol. 2007;89:279-84.
34. De Marco A, Feitosa AM, Gomes MM, Parente GB, Victor EG. Pressão de pulso obtida por monitorização residencial da pressão

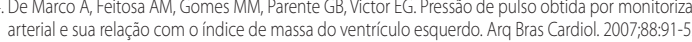

35. Machado RC, Branco JNR, Michel JLM, Gabriel EA, Locali RF, Helito RAB, et al. Caracterizaçăo dos cuidadores de candidatos a Thachado RC, Branco JNR, Michel JLM, Gabriel EA, Locali RF, Helito RAB, et al . Ca
transplante do coraçấo na Unifesp. Rev Bras Cir Cardiovasc. 2007;22:432-40.

36. Cressoni Avanci LE, Braile DM, Lima-Oliveira APM, Taboga SR, Martins AS, et al . Efeitos das cardioplegias sangüinea e cristalóide no miocárdio hipertrófico de coelho: avaliaçăo estrutural e ultra-estrutural. Rev Bras Cir Cardiovasc. 2007;22:24-32.

37. Zornoff LAM, Duarte DR, Minicucci MF, Azevedo PS, Matsubara BB, Matsubara LS, et al. Efeitos do betacaroteno e do tabagismo sobre a remodelaçăo cardíaca pós-infarto do miocárdio. Arq Bras Cardiol. 2007; 89:151-7.

38. Dinkhuysen JJ, Andrade AJP, Manrique R, Saito CSM, Leme J, Biscegli F. Bomba sangüínea espiral: concepção, desenvolvimento e aplicaçăo clínica de projeto original. Rev Bras Cir Cardiovasc. 2007;22:218-23.

39. Silveira Filho LM, Petrucci Junior O, Carmo MR, Oliveira PPM, Vilarinho KAS, Vieira RW, et al . Padronização de modelo de coração isolado "working heart" com circulação parabiótica. Rev Bras Cir Cardiovasc. 2008;23:14-22

40. Fonseca SMS, Belo LG, Carvalho H, Araújo N, Munhoz C, Siqueira L et al. Acompanhamento clínico de pacientes portadores de cardioversor-desfibrilador implantável. Arq Bras Cardiol. 2007;88:8-16.

41. Nunes FTB, Conforti-Froes NDT, Negrelli WF, Souza DRS. Fatores genéticos e ambientais envolvidos na degeneração do disco intervertebral. Acta Ortop Bras. 2007;15:9-13.

42. Oliveira VM, Puertas EB, Alves MTS, Yamashita HK. Estudo comparativo dos mecanorreceptores dos discos intervertebrais normais e degenerados da coluna lombar de humanos pela radiografia, ressonância magnética e estudo anatomopatológico. Acta Ortop Bras. 2007;15:35-9.

43. Demange MK, Kakuda CMS, Pereira CAM, Sakaki MH, Albuquerque RFM. Influência do ligamento da cabeça do fêmur na mecânica do quadril. Acta Ortop Bras. 2007;15:187-90.

44. Bispo Júnior RZ, Kawano CT, Guedes AV. Lesões crônicas múltiplas dos ligamentos do joelho: análise epidemiológica de mais de cem casos. Clinics. 2008:63:3-8.

45. Satomi E, Teodoro WR, Parra ER, Fernandes TD, Velosa APP, Capelozzi VL, et al . Alteraçōes na distribuiçăo histoanatômica de colágenos tipo I, III e V promovem remodelaçăo adaptativa na ruptura do tendão tibial posterior. Clinics. 2008:63:9-14.

46. Hamra A, Ribeiro MB, Miguel OF. Correlação entre fratura por queda em idosos e uso prévio de medicamentos. Acta Ortop Bras. 2007;15:143-5

47. Belloti JC, Santos JBG, Atallah AN, Albertoni WM, Faloppa F. Fraturas do rádio distal (Fratura de Colles). Sao Paulo Med J. 2007;125:132-8.

48. Fernandes PM, Sabino Neto M, Veiga DF, Abla LEF, Mundim CDA, Juliano Y, et al. Dores na coluna: avaliação em pacientes com hipertrofia mamária. Acta Ortop Bras. 2007;15:227-30

49. Yamamoto PA, Lahoz GL, Takata ET, Masiero D, Chamlian TR. Avaliação da função e qualidade de vida em pacientes submetidos a artroplastia de ressecção tipo Girdlestone. Acta Ortop Bras. 2007;15:214-7.

50. Makdisse M, Nascimento Neto R, Chagas ACP, Brasil D, Borges JL, Oliveira A, et al . Versão em português, adaptaçăo transcultural e validaçăo do Questionário de Claudicaçăo de Edimburgo. Arq Bras Cardiol. 2007;88:501-6.

51. Coelho FAC, Moutinho MAE, Miranda VA, Tavares LR, Rachid M, Rosa MLG, et al. Associaçăo da síndrome metabólica e seus componentes na insuficiência cardíaca encaminhada da atençăo primária. Arq Bras Cardiol. 2007;89:42-51.

52. Velásquez-Meléndez G, Gazzinelli A, Côrrea-Oliveira R, Pimenta AMl, Kac G. Prevalência da síndrome metabólica em área rural do Brasil. Sao Paulo Med J. 2007:125:155-62

53. Nakazone MA, Pinheiro A, Braile MCVB, Pinhel MAS, Sousa GF Pinheiro Júnior S, et al .Prevalência de síndrome metabólica em indivíduos brasileiros pelos critérios de NCEP-ATPIII e IDF. Rev Assoc Med Bras. 2007:53:407-13.

54. Karnikowski M, Córdova C, Oliveira RJ, Karnikowski MGO, Nóbrega OT. Esteatose hepática não-al cóolica e síndrome metabólica em brasileiros idosos e de meia-idade. Sao Paulo Med J. 2007:125:333-7.

55. Greve JAA Bordini ACPG Camanho GL Correlação entre índice de massa corpórea e equilibrio postural Clinics, 2007:62:717-20

56. Fernandes RA, Rosa CSC, Silva CB, Bueno DR, Oliveira AR, Freitas Júnior IF. Desempenho de diferentes valores críticos de índice de massa corporal na identificaçăo de excesso de gordura corporal e obesidade abdominal em adolescentes. Rev Assoc Med Bras. 2007;53:515-9.

57. Rosa MLG, Mesquita ET, Rocha ERR, Fonseca VM. Indice de massa corpóreara e circunferência de cintura como marcadores de hipertensão em adolescentes. Arq Bras Cardiol. 2007;88:573-8.

58. Araújo F, Yamada AT, Markus MRP, Antelmi I, Latorre MRDO, Mansur AJ. Aumento do índice de massa corporal em relação a variáveis clínicas e laboratoriais quanto ao sexo em indivíduos sem evidências de cardiopatia. Arq Bras Cardiol. 2007;88:624-9. 59. Nunes MMA, Figueiroa JN, Alves JGB. Excesso de peso, atividade física e hábitos alimentares entre adolescentes de diferentes classes econômicas em Campina Grande (PB). Rev Assoc Med Bras. 2007:53:130-4.

60. Barreto ACNG, Brasil LMP, Maranhão HS. Sobrepeso: uma nova realidade no estado nutricional de pré-escolares de Natal, RN. Rev Assoc Med Bras. 2007:311-6.

61. Magnani KL, Cataneo AJM. Força dos músculos respiratórios em obesos e influência da distribuiçăo do tecido adiposo. Sao

Paulo Med J 2007.25.215-9.

62. Rocha IEGM, Victor EG, Braga Barbosa AR, Souza JMP, Leb

Med Bras. 2007:53:75-9.

64. Guedes DP, G Assoc Med Bras. 2007;53:59-63.

65. Gomes WJ, Mendonça JT, Braile DM. Resultados em cirurgia cardiovascular: oportunidade para rediscutir o atendimento médico e cardiológico no sistema público de saúde do país. Rev Bras Cir Cardiovasc. 2007;22:-IV.

66. Grieger MCA. Escritores-fantasma e comércio de trabalhos científicos na internet: a ciência em risco. Rev Assoc Med Bras. 2007;53:247-51

67. Galvão FHF, Caires RA, Azevedo-Neto RS, Mory EK, Figueira ERR, Otsuzi TS, et al. Conhecimento e opinião de estudantes de medicina sobre doaçăo e transplante de órgăos. Rev Assoc Med Bras. 2007:53:401-6.

68. Gawryszeski VP. Um retrato das mortes por causas externas no Estado de Săo Paulo, 2003. Sao Paulo Med J. 2007;125:139-43. 69. Pugliese R, Zanella MT, Blay SL, Plavinik F, Andrade MA, Galväo R. Eficácia de uma intervençăo psicológica no estilo de vida para reduçäo do risco coronariano. Arq Bras Cardiol. 2007:89:225-30.

70. Sogame LCM, Faresin SM, Vidotto MC, Jardim JR. Estudo pós-operatório da capacidade vital e ventilação em craniotomia eletiva. Sao Paulo Med J. 2008:126:11-6.

71. Maciel AT, Park M. Ânions nẫo mensuráveis são responsáveis pela maior parte da acidose metabólica de pacientes com hiperlactatemia. Clinics. 2007:62:55-62.

72. Moser AM, Reggiani C, Urbanetz A. Comportamento sexual de risco entre estudantes universitárias dos cursos de ciências da saúde. Rev Assoc Med Bras. 2007;53:116-21.

73. Antonello VS, Antonello ICF, Santos CA. Sensibilidade gustativa ao sal, natriúria e pressão arterial em indivíduos normotensos. Rev Assoc Med Bras. 2007;53:142-6. 